\title{
Isoquercetin activates the ERK1/2-Nrf2 pathway and protects against cerebral ischemia-reperfusion injury in vivo and in vitro
}

\author{
MIAO CHEN*, LI-HUA DAI* ${ }^{*}$ AIHUA FEI, SHU-MING PAN and HAI-RONG WANG \\ Department of Emergency, Xinhua Hospital Affiliated to Shanghai Jiaotong University, \\ Shanghai 200092, P.R. China
}

Received June 18, 2015; Accepted June 8, 2016

DOI: $10.3892 /$ etm.2017.4093

\begin{abstract}
Isoquercetin has exhibited a wide range of therapeutic properties, including antioxidant, anti-inflammatory and anti-allergic activities. The aim of the present study was to investigate the effect of isoquercetin on rats with $2 \mathrm{~h}$ middle cerebral artery occlusion (MCAO) and evaluate the neuroprotective effect of isoquercetin on a primary culture of rat hippocampal neuronal cells subjected to oxygen-glucose deprivation followed by reoxygenation (OGD/R). In vivo, the rats treated with isoquercetin exhibited a lower degree of neurological dysfunction and smaller infarct volume than the vehicle-treated rats. In vitro, it was found that isoquercetin prevented the OGD/R-induced increase in apoptosis, lactate dehydrogenase release and reduction in cell viability. Additionally, isoquercetin induced the upregulation of nuclear factor erythroid 2-related factor 2 gene and protein expression, and increased extracellular signal-regulated kinase 1 and 2 (ERK1/2) phosphorylation. This indicates that the ERK1/2 pathway may contribute to the neuroprotective effect of isoquercetin against OGD/R-induced oxidative damage in rat hippocampal neurons. These findings suggest the potential importance of isoquercetin in the treatment of ischemia/reperfusion-related brain injury and associated diseases.
\end{abstract}

\section{Introduction}

Ischemic stroke is a major threat to health and a significant cause of human mortality. Thus, understanding the pathogenesis

Correspondence to: Professor Hai-Rong Wang or Professor Shu-Ming Pan, Department of Emergency, Xinhua Hospital Affiliated to Shanghai Jiaotong University, 1665 Kongjiang Road, Shanghai 200092, P.R. China

E-mail: hairongwang201503@163.com

E-mail: shumingpan2014@163.com

*Contributed equally

Key words: isoquercetin, middle cerebral artery occlusion, nuclear factor erythroid 2-related factor 2, extracellular signal-regulated kinases 1 and 2, antioxidant of ischemic stroke and the development of strategies that may prevent it is (1). Ischemic stroke, in which a blood vessel is suddenly occluded by a thrombus or embolism, accounts for $87 \%$ of all cases of stroke (2). Ischemia and reperfusion induce cellular damage, which triggers a cascade of biochemical processes that ultimately induce structural and functional changes in organs or tissues, with transient global brain ischemia causing selective neuronal injury (3).

As a regular supply of oxygen and glucose is essential for the maintenance of normal neuronal functions, the application of oxygen-glucose deprivation (OGD) to cultured hippocampal tissue can be used to create a cellular model of ischemic stroke. The lack of a regular supply of oxygen and glucose, even for a short period, results in ischemia/re-oxygenation and the production of reactive oxygen species (ROS), culminating in neuronal cell death and brain damage (4).

Isoquercetin is a dietary flavonoid present in a variety of medicinal and dietary plants, including vegetables, herbs and flowers (5). Isoquercetin has been shown to have numerous therapeutic properties, including anti-inflammatory, antioxidant and anti-allergic activities (6). Morand et al (7) demonstrated that isoquercetin was better absorbed than quercetin and had higher bioavailability. However, to the best of our knowledge, whether isoquercetin has the ability to reduce infarct volumes and behavioral deficits has not been studied previously. In the present study, the effects of isoquercetin on transient middle cerebral artery occlusion (MCAO) and primary hippocampal neurons subjected to OGD followed by reoxygenation (OGD/R), which are models commonly used for examining the molecular mechanisms of ischemic stroke injury in vivo and in vitro, were investigated. In addition, the ability of isoquercetin to activate the extracellular signal-regulated protein kinase 1 and 2 (ERK1/2) nuclear factor erythroid 2-related factor 2 (Nrf2) pathway in vivo and in vitro was evaluated in order to further elucidate the precise protective mechanisms.

\section{Materials and methods}

Animals and MCAO surgery. A total of 608 -week old male Sprague-Dawley (SD) rats (220-260 g) were obtained from the Experimental Animal Center of Xinhua Hospital (Shanghai, China). All animals were housed and handled according to guidelines from the Institutional Animal Care and Use Committee of Xinhua Hospital and all animal experiments were approved 
by the Shanghai Jiaotong University committee. The rats were randomly allocated to one of four groups: Control, sham, model and isoquercetin. In the model group, rats were anesthetized via the intraperitoneal injection of $10 \%$ chloral hydrate. The rectal temperature was monitored and maintained at $37.0 \pm 0.5^{\circ} \mathrm{C}$ by the use of a feedback-regulated heating system during surgery. Permanent focal ischemia was induced by occluding the right middle cerebral artery (MCA) via an intraluminal technique, as previously described (8). Briefly, a 4-0 nylon monofilament suture with a slightly enlarged rounded tip was inserted into the stump of the external carotid artery and advanced into the lumen of the internal carotid artery until it reached and occluded the MCA. The distance from the bifurcation of the common carotid artery (CCA) to the tip of the inserted suture averaged $18-20 \mathrm{~mm}$. Sham operated animals were subjected to the aforementioned procedures, with the exception of suture insertion. Rats in the isoquercetin group were subjected to MCAO surgery and treated with intravenous $50 \mathrm{mg} / \mathrm{kg}$ isoquercetin (Dalian Institute of Chemical Physics, Chinese Academy of Sciences, Dalian, China) once a day for 7 days. Rats that did not undergo any surgery served as the control group.

Neurological function and measurement of infarct volume. Neurological deficits were scored by double-blind testing based on a modified Longa EZ test (9), on a scale from 0-5 in which 0 represented no deficit, 4 indicated the maximal deficit and 5 indicated mortality $24 \mathrm{~h}$ following MCAO. Results were statistically analyzed using the rank sum test. At $24 \mathrm{~h}$ after ischemia, brains were removed and sliced into 5 coronal sections $2 \mathrm{~mm}$ thick (10). Sections were immediately stained with $1 \%$ tertrazolium chloride at $37^{\circ} \mathrm{C}$ for $30 \mathrm{~min}$ as previously described (11). The infarct volume was expressed as a percentage of the volume of the total brain.

Primary hippocampalneuron cell cultures. All experiments were approved by the Institutional Animal Care and Use Committee of Xinhua Hospital and according to guidelines developed by China Council on Animal Care. A total of 20 male newborn SD rats $(<24 \mathrm{~h}$ old, mean weight, $5 \pm 2 \mathrm{~g}$ ) were purchased from the SLAC Laboratory Animal Center of Shanghai (China). Primary hippocampal neuron cultures were established from the cerebral hippocampi of newborn SD rats. Newborn rats were sacrificed by cervical dislocation and brains were cleaned of meninges and blood vessels. The entire cerebral hippocampus was isolated and cells were dissociated by treatment with $0.25 \%$ trypsin solution for $10 \mathrm{~min}$ at $37^{\circ} \mathrm{C}$. Subsequently, $10 \%$ fetal bovine serum (Gibco; Thermo Fisher Scientific, Inc., Waltham, MA, USA) was added and the dissociated cells were forced through a 300 mesh. Following centrifugation (1,000 x g, 5 min), hippocampal neurons were resuspended and plated in six-well plates coated with poly-D-lysine (Sigma-Aldrich; Merck Millipore, Darmstadt, Germany) and grown in Neurobasal media (Gibco; Thermo Fisher Scientific, Inc.) with $500 \mu \mathrm{M}$ glutamine and $2 \%$ B27 supplement (both Gibco; Thermo Fisher Scientific, Inc.) in a humidified incubator with $5 \% \mathrm{CO}_{2}$ at $37^{\circ} \mathrm{C}$. The cell culture medium was replaced with fresh culture medium on days 3 and 5 in vitro.

OGD/R culture method. Primary hippocampal neurons were placed in an anaerobic chamber (HERAcell 150 incubator;
Thermo Fisher Scientific, Inc.) and the partial oxygen pressure was maintained at $<2 \mathrm{mmHg}$ ). The medium was replaced with a pre-warmed $\left(37^{\circ} \mathrm{C}\right)$ glucose-free balanced salt solution. An anaerobic gas mixture comprising $95 \% \mathrm{~N}_{2}$ and $5 \% \mathrm{CO}_{2}$ was bubbled through the solution for $30 \mathrm{~min}$. Cell cultures subjected to OGD were incubated in the solution at $37^{\circ} \mathrm{C}$ for different time periods to produce oxygen deprivation and then re-oxygenated (returned to the normal aerobic environment). Experimental parameters were assayed $4 \mathrm{~h}$ following re-oxygenation. The primary cultures of rat hippocampal neurons were pretreated with 25,50 and $100 \mu \mathrm{g} / \mathrm{ml}$ isoquercetin for $24 \mathrm{~h}$, followed by exposure to OGD for $4 \mathrm{~h}$, then reperfusion for $24 \mathrm{~h}$. The hippocampal neurons cultured in plain medium served as control. At the end of cell treatments, different tests were carried out as described below. PD98059 (HY-12028; MCE, Monmouth Junction, NJ, USA) was the inhibitor of the MEK.

Cell viability assay. The viability of the neurons in the five in vitro treatment groups was determined using a 3-(4,5-dimethylthiazol-2-yl)-2,5-diphenyltetrazolium

bromide (MTT) assay. Hippocampal neurons were seeded onto 96 -well plates $\left(1 \times 10^{4}\right.$ cells/well). Following treatment, $0.2 \mathrm{mg} / \mathrm{ml}$ MTT salt (Sigma-Aldrich; Merck Millipore) was added to each well and the cells were further incubated in $5 \% \mathrm{CO}_{2}$ at $37^{\circ} \mathrm{C}$ for $4 \mathrm{~h}$. Dimethyl sulfoxide was then added to dissolve the formazan crystals for $20 \mathrm{~min}$. The number of viable cells was assessed by measurement of the absorbance at $490 \mathrm{~nm}$ using a Safire 2 microplate reader (Tecan, San Jose, CA, USA).

Measurement of ROS production. ROS production in the five in vitro treatment groups was measured using the ROS-sensitive dye 2',7'-dichlorofluorescein diacetate ( $\mathrm{H}_{2} \mathrm{DCFDA}$; Invitrogen; Thermo Fisher Scientific, Inc.) using the protocols provided by the manufacturer. In the presence of ROS, $\mathrm{H}_{2} \mathrm{DCF}$ is rapidly oxidized to form highly fluorescent DCF. Hippocampal neurons were incubated with $\mathrm{H}_{2}$ DCFDA $(10 \mu \mathrm{M})$ for $30 \mathrm{~min}$ at $37^{\circ} \mathrm{C}$. Fluorescence images were obtained using a fluorescence microscope (BX-51; Olympus Corporation, Tokyo, Japan). The fluorescence intensity was measured using Image J software (National Institutes of Health, Bethesda, MD, USA), averaged and normalized to the control value.

Lactate dehydrogenase $(\mathrm{LDH})$ release assay. Cytotoxic activity in the five in vitro treatment groups was determined using an LDH release assay as described previously (12). Briefly, LDH in the culture medium (released LDH) was quantified using an LDH cytotoxicity detection kit (Nanjing Jiancheng Biotech, Nanjing, China) according to the protocol provided by the manufacturer. The relative amount of released LDH was used to reflect the extent of cell injury.

Apoptosis assay by flow cytometry. Fluorescein isothiocyanate (FITC)-Annexin V and propidium iodide (PI) double staining was used to examine the OGD/R-induced apoptosis of the hippocampal neurons in the five in vitro treatment groups. The assay was conducted using an FITC-Annexin V/PI apoptosis detection kit according to the manufacturer's protocol (Invitrogen; Thermo Fisher Scientific, Inc.). Briefly, 
neurons were cultured in 6 -well plates $\left(1 \times 10^{6}\right.$ cells/well $)$ and treated as described above. Cells were collected after OGD/R and flow cytometry was conducted to assess the apoptosis of the cells.

RNA isolation and reverse transcription-quantitative polymerase chain reaction ( $R T-q P C R)$. Total RNA was isolated from cells from each group using TRIzol reagent (Invitrogen; Thermo Fisher Scientific, Inc.) and treatment of RNA was performed using DNase (Takara Biotechnology, Co., Ltd., Dalian, China). Reverse transcription was performed using an Omniscript RT kit (Promega Corporation, Madison, WI, USA) according to the manufacturer's protocol. qPCR was subsequently performed to determine gene expression using the SYBR green Master Mix (Takara Biotechnology, Co., Ltd., Dalian, China). The primers for Nrf2 were: Forward, 5'-TACTCCCAGGTTGCCCACA-3' and reverse, 5'-CAT CTACAAACGGGAATGTCTG-3'. The primers for ERK were: Forward, 5'-GAGGCAGCAGGAACAATGCT-3' and reverse, 5'-CCAGCTTTCTGCAGAGGGAA-3'. The primers for glyceraldehyde 3-phosphate dehydrogenase (GAPDH) were: Forward, 5'-CATCTTCCAGGAGCGAGACC-3' and reverse, 5'-CTCGTGGTTCACACCCATCA-3'. The expression level of each target mRNA relative to GAPDH was calculated on the basis of the threshold cycle (CT) as $\mathrm{r}=2-\Delta(\Delta \mathrm{CT})(13)$.

Western blot analysis. Hippocampal neurons were washed twice with ice-cold phosphate-buffered saline and then lysed with ice-cold lysis buffer (20 mM Tris- $\mathrm{HCl}(\mathrm{pH}=7.5)$, $150 \mathrm{mM} \mathrm{NaCl}, 1 \mathrm{mM}$ Na3VO4, 1 mM PMSF, 1 mM EDTA, $1 \% \mathrm{NP} 40,50 \mathrm{mM} \mathrm{NaF}$ ) for $30 \mathrm{~min}$. Cell lysates were centrifuged at $10,000 \times \mathrm{g}$ for $15 \mathrm{~min}$ at $4^{\circ} \mathrm{C}$. Cell lysates were separated by sodium dodecyl sulfate-polyacrylamide $12 \%$ gel electrophoresis and transferred to polyvinylidene fluoride membranes. Following incubation with blocking buffer (Tris-buffered saline $+0.1 \%$ Tween-20 $($ TBST) $+5 \%$ non-fat milk), membranes were probed with rabbit anti-Nrf2 polyclonal antibody, (1:1,000, ab31163 abcam, Cambridge, UK) mouse anti-ERK1+ERK2 polyclonal antibody (1:1,000, ab54230, abcam), rabbit anti-ERK1 (phospho T202) + ERK2 (phospho T185) antibody (1:1,000, ab201015, abcam) and mouse anti-GAPDH monoclonal antibody $(1: 2,000$, ab8245, abcam) overnight at $4^{\circ} \mathrm{C}$. The membranes were then washed with TBST and incubated with a horseradish peroxide-conjugated secondary antibody (1:1,000, 00001-2, ProteinTech Group, Inc., Chicago, IL, USA), and signals were detected with an enhanced chemiluminescent reagent (EMD Millipore, Billerica, MA, USA). Images were captured using a Bio-Rad VersaDoc 3000 (Bio-Rad Laboratories, Inc., Gladesville, NSW, Australia) and protein levels were quantified using Image-pro Plus 6.0 (Media Cybernetics, MD Rockville, USA).

Statistical analysis. Data are presented as the mean \pm standard error of the mean of at least three independent preparations. Statistical analysis was conducted by analysis of variance with Tukey's or Scheffé's post hoc tests using SPSS statistical analysis software, version 17.0 (SPSS, Inc., Chicago, IL, USA). $\mathrm{P}<0.05$ was considered to indicate a statistically significant difference.

\section{Results}

Isoquercetin protects against brain injury by transient $M C A O$. The potential ability of isoquercetin to protect against transient MCAO-induced damage was investigated. Results showed that infarct volume was $41.05 \pm 4.19 \%$ in the model group and $21.35 \pm 2.03 \%$ in the $50 \mathrm{mg} / \mathrm{kg}$ isoquercetin group. Statistical analysis revealed that infarct volume in the $50 \mathrm{mg} / \mathrm{kg}$ isoquercetin group was significantly smaller than that of the model group $24 \mathrm{~h}$ after ischemia $(\mathrm{P}<0.01$; Fig. 1A and $\mathrm{B}$ ). Furthermore, $50 \mathrm{mg} / \mathrm{kg}$ isoquercetin improved the neurological function compared with that of the model group at 7 days after ischemia (Fig. 1C).

Isoquercetin increases Nrf2 expression in the hippocampus in vivo. In the hippocampal tissue, the expression of Nrf 2 at the mRNA and protein levels was decreased in the model group 7 days after ischemia, and the reduction in expression levels was significantly attenuated in the isoquercetin treated-group (Fig. 2).

Isoquercetin protects primary culture of rat hippocampus neurons against $O G D / R$-induced cytotoxicity with attenuation of $L D H$ release and $R O S$ content. An MTT assay, in which MTT was reduced by mitochondrial reductase in viable cells, was used to determine cell viability. Cultured hippocampal neurons were pre-conditioned with isoquercetin for $24 \mathrm{~h}$ prior to $4 \mathrm{~h}$ OGD and $24 \mathrm{~h}$ normoxia with isoquercetin for recovery. As shown in Fig. 3A, isoquercetin at concentrations of 25, 50 and $100 \mu \mathrm{g} / \mathrm{ml}$ significantly promoted the cell viability of cultured hippocampus neurons. The protective activity of isoquercetin against OGD/R-induced injury in cultured hippocampus neurons was further investigated using ROS and LDH assays. In these assays, 25,50 or $100 \mu \mathrm{g} / \mathrm{ml}$ isoquercetin was added to the culture medium from $24 \mathrm{~h}$ prior to OGD until the end of recovery. The results shown in Fig. $3 \mathrm{~B}$ and $\mathrm{C}$ reveal that OGD/R significantly increased the ROS content of and LDH release by hippocampal neurons compared with those in the control group. Furthermore, LDH release and ROS content were significantly higher in the group treated with $100 \mu \mathrm{g} / \mathrm{ml}$ isoquercetin compared with the other treatment groups.

FITC-Annexin V/PI double staining. As shown in Fig. 4, almost no apoptotic cells were detected in the control group, but a large number were present in the OGD/R-treated group. Treatment of the cells with isoquercetin at doses of 25,50 and $100 \mu \mathrm{g} / \mathrm{ml}$ significantly reduced the proportion of apoptotic cells in a concentration-dependent manner, indicating the ability of isoquercetin to inhibit an early event of the apoptotic process.

Isoquercetin upregulates the gene and protein expression of Nrf 2 and triggers ERK1/2 phosphorylation. As shown in Fig. 5A and B, the expression of Nrf2 at the mRNA and protein levels following treatment with 25,50 or $100 \mu \mathrm{g} / \mathrm{ml}$ isoquercetin for $24 \mathrm{~h}$ in cells that had undergone OGD/R was evaluated using RT-qPCR and western blotting, respectively. The protein expression of Nrf2 in hippocampal neurons in the $100 \mu \mathrm{g} / \mathrm{ml}$ group increased by $200 \%$ compared with the OGD/R group. This increase in Nrf2 protein expression was 

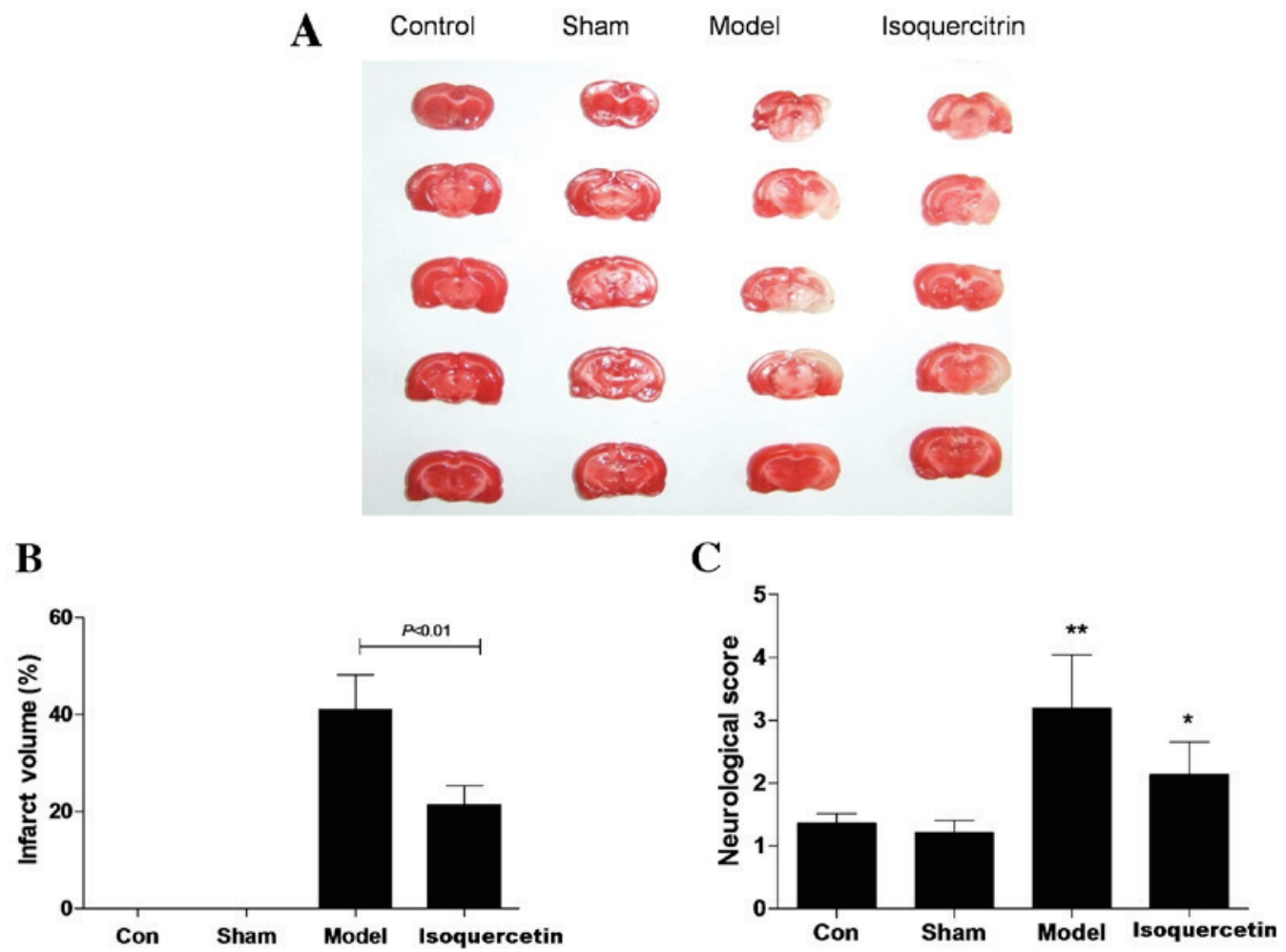

Figure 1. (A) Representative images of triphenyltetrazolium chloride-stained sections after ischemia. (B) Infarct volumes in the control (Con; $\mathrm{n}=8)$, sham ( $\mathrm{n}=8$ ), middle cerebral artery occlusion model $(\mathrm{n}=12)$ and isoquercetin $50 \mathrm{mg} / \mathrm{kg}(\mathrm{n}=12)$ groups after completion of the model. (C) Neurological scores determined at 7 days after ischemia. In (B) and (C), data are presented as the mean \pm standard error of the mean. ${ }^{*} \mathrm{P}<0.05$ and ${ }^{* *} \mathrm{P}<0.01$ vs. the control group.
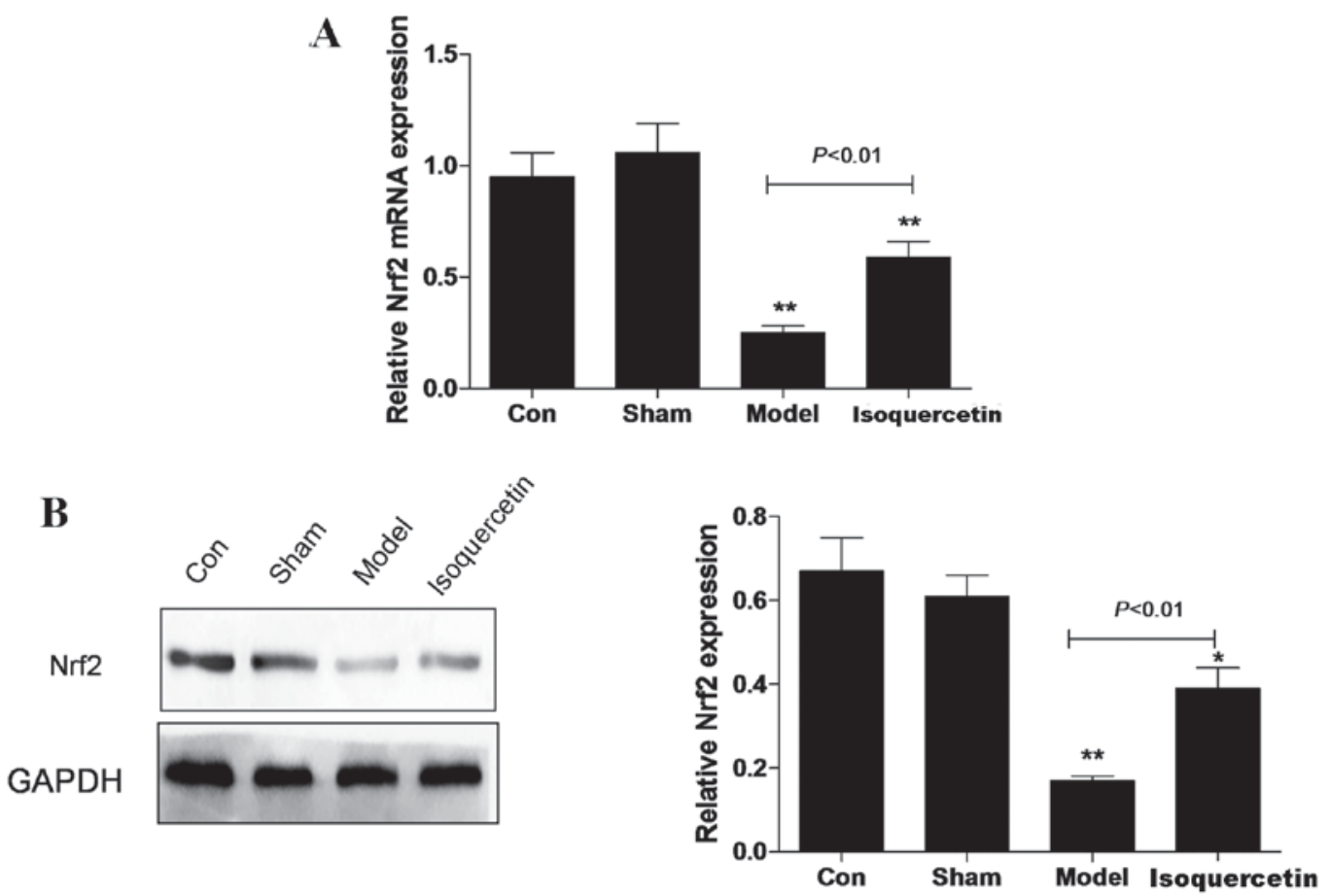

Figure 2. mRNA and protein expression of Nrf2 in the hippocampal tissue of middle cerebral artery occlusion model animals after isoquercetin treatment were examined by (A) reverse transcription-quantitative polymerase chain reaction and (B) western blotting, respectively. GAPDH was used as an internal control. Data are shown as the mean \pm standard error of the mean from three independent experiments. ${ }^{*} \mathrm{P}<0.05$ and ${ }^{* *} \mathrm{P}<0.01$ vs. the control group. Nrf2, nuclear factor erythroid 2-related factor 2; GAPDH, glyceraldehyde 3-phosphate dehydrogenase.

attenuated in the presence of PD98059, which suggests that the Nrf2-related neuroprotective effect of isoquercetin may be mediated via the ERK1/2 signaling pathway (Fig. 5C and D).
Western blotting results show that OGD/R slightly induced ERK1/2 phosphorylation. Isoquercetin increased ERK1/2 phosphorylation and $\mathrm{Nrf} 2$ expression levels in OGD/R-treated 

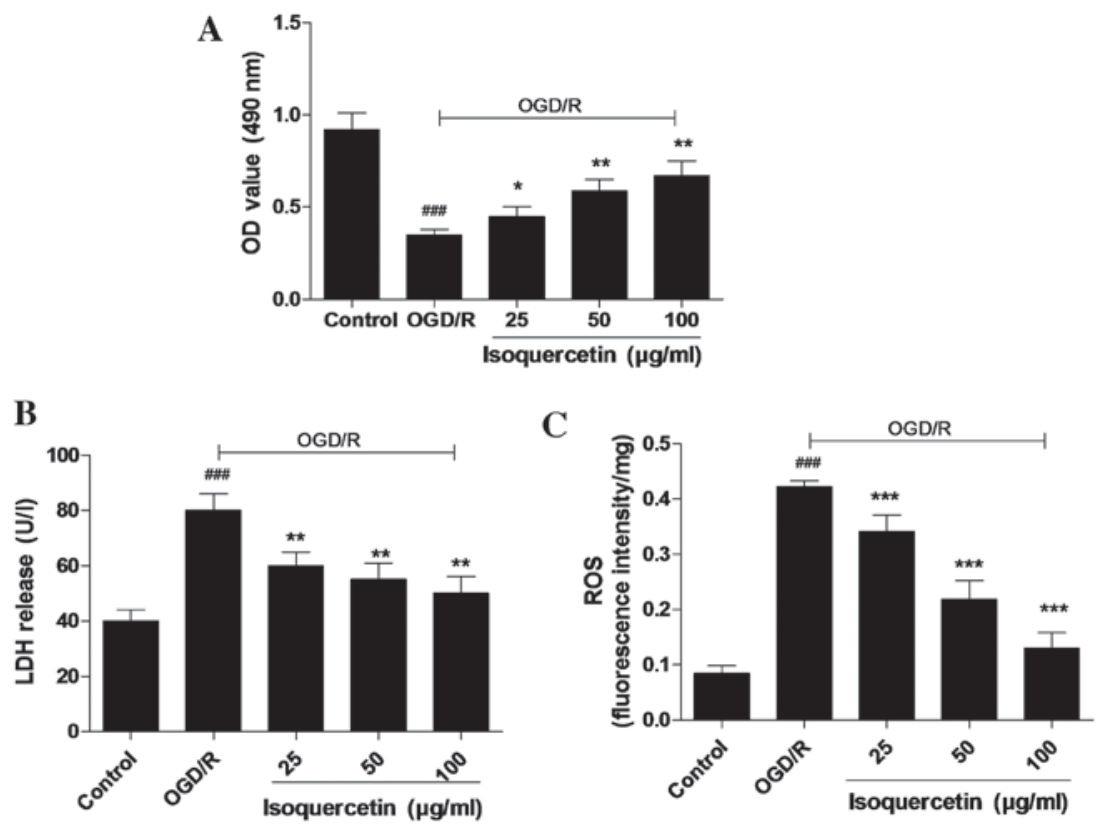

Figure 3. Effects of different concentrations of isoquercetin on primary cultures of rat hippocampal neurons after OGD/R. (A) Cell viability was determined by MTT assay, and (B) LDH release and (C) ROS levels were measured. Data are shown as the mean \pm standard error of the mean $(\mathrm{n}=6)$. ${ }^{*} \mathrm{P}<0.05$, ${ }^{* * *} \mathrm{P}<0.01$ and ${ }^{* * * *} \mathrm{P}<0.001$ vs. the $\mathrm{OGD} / \mathrm{R}$ group; ${ }^{\# \# \#} \mathrm{P}<0.001$ vs. the control group. OGD/R, oxygen-glucose deprivation followed by reoxygenation; MTT, 3-(4,5-dimethylthiazol-2-yl)-2,5-diphenyltetrazolium bromide; LDH, lactate dehydrogenase; ROS, reactive oxygen species; OD, optical density.

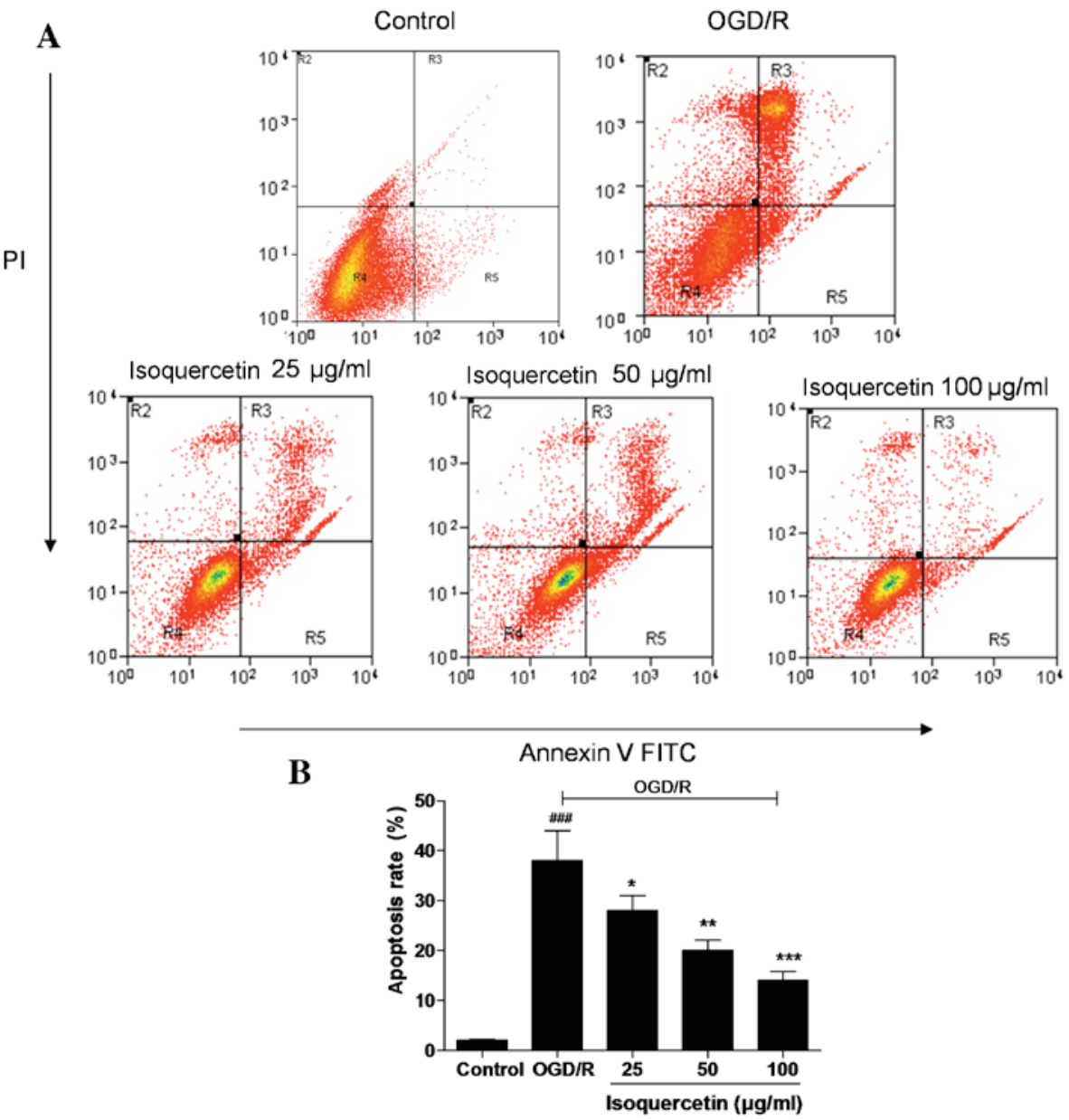

Figure 4. Effects of different concentrations of isoquercetin on the OGD/R-induced apoptosis of hippocampal neurons determined by the Annexin V/PI flow cytometric assay method, which can detect cells at an early stage of the apoptotic pathway and is able to distinguish between apoptotic and necrotic cells. (A) Images shown are representative of three experiments. (B) Apoptosis rates, presented as mean \pm standard error of the mean $(n=3)$. ${ }^{*} \mathrm{P}<0.05$, ${ }^{* *} \mathrm{P}<0.01$ and ${ }^{* * * *} \mathrm{P}<0.001$ vs. the $\mathrm{OGD} / \mathrm{R}$ group; ${ }^{\# \# "} \mathrm{P}<0.001$ vs. the control group. $\mathrm{OGD} / \mathrm{R}$, oxygen-glucose deprivation followed by reoxygenation; PI, propidium iodide; FITC, fluorescein isothiocyanate. 
A

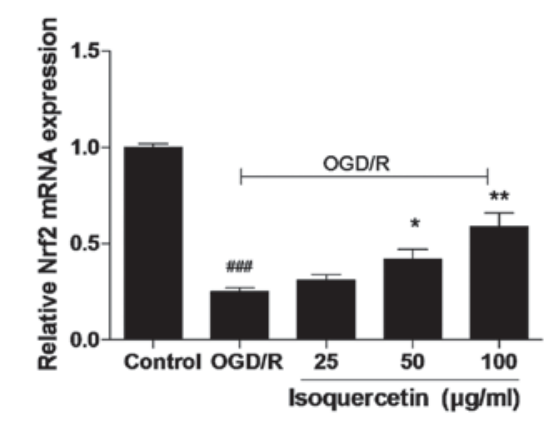

C

$\begin{array}{cccccc}\text { PD98059 } & 1 & 2 & 3 & 4 & 5 \\ \text { Isoquercetin } & - & - & - & + & + \\ \text { OGD/R } & - & + & + & - & + \\ & & & + & +\end{array}$

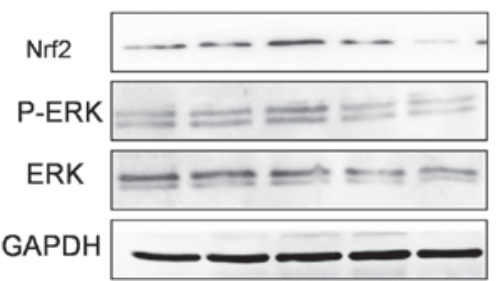

B

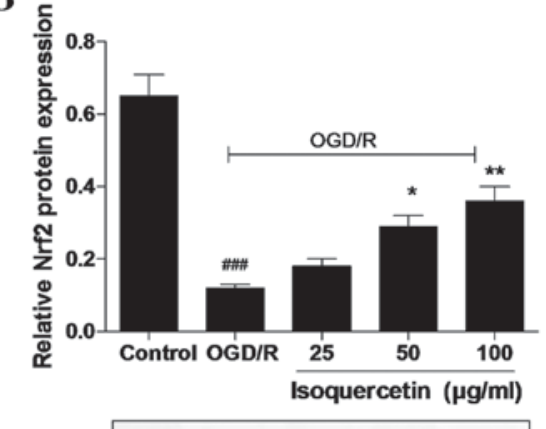

$\mathrm{Nrf2}$

GAPDH

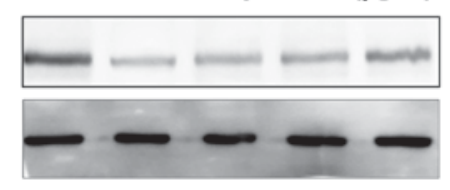

D

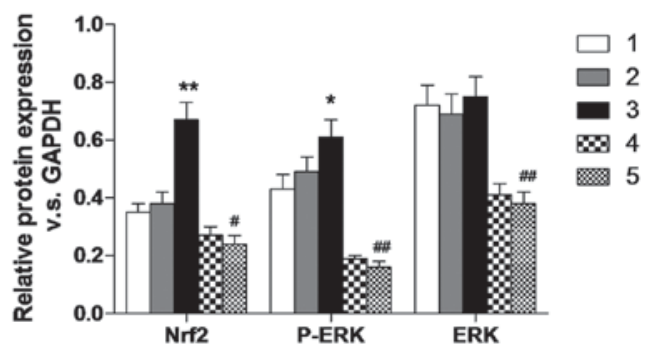

Figure 5. Expression of Nrf2 at the mRNA and protein levels was determined by (A) reverse transcription-quantitative polymerase chain reaction (RT-qPCR) and (B) western blotting, respectively. Isoquercetin increases the phosphorylation of ERK in primary hippocampal neurons. (C) Hippocampal neuron lysates were subjected to western blotting to evaluate Nrf2 levels and the phosphorylation of ERK in the presence and absence of PD98059. (D) The densities of bands representing phosphorylated ERK1/2 and Nrf2 were quantified by densitometric scanning from three independent experiments. GAPDH was used as an internal control. Representative western blots are shown for samples run in duplicate from one of three independent experiments. Graphical data are presented as mean \pm standard error of the mean $(\mathrm{n}=3)$. ${ }^{*} \mathrm{P}<0.05$ and ${ }^{* *} \mathrm{P}<0.01$ vs. the $\mathrm{OGD} / \mathrm{R}$ group; ${ }^{\#} \mathrm{P}<0.05,{ }^{\# \#} \mathrm{P}<0.01$ and ${ }^{\# \# \#} \mathrm{P}<0$. vs. the control group. Nrf2, nuclear factor erythroid 2-related factor 2; ERK, extracellular signal-regulated protein kinase 1 and 2; GAPDH, glyceraldehyde 3-phosphate dehydrogenase; OGD/R, oxygen-glucose deprivation followed by reoxygenation.

cells by 29 and $91 \%$ compared with those in cells treated with $\mathrm{OGD} / \mathrm{R}$ alone. The isoquercetin-induced increases in ERK1/2 phosphorylation and Nrf2 levels were completely abrogated in the presence of PD98059.

\section{Discussion}

Diets rich in colorful, flavonoid-rich fruits, vegetables and nuts have been shown to improve the health of the brain by reducing oxidative stress, and improving neuronal signaling, behavior and cognitive functions (14). The results of the present study demonstrated for the first time that intravenous post-treatment with isoquercetin had a protective effect against transient MCAO-induced brain damage in rats, with positive effects on neurobehavioral function, infarct volume and neuronal loss without any adverse effects on physiological parameters.

Oxidative stress has been shown to be a key cause of neurodegenerative and vascular diseases pathologies affecting the brain $(15,16)$. Consistent with this, the present study found that isoquercetin could downregulate oxidative damage in primary hippocampal neurons subjected to $\mathrm{OGD} /$ reoxygenation in vitro. Also, isoquercetin reduced the amount of cell death induced by $\mathrm{OGD} / \mathrm{R}$ in a dose-dependent manner. The results of the present indicate that isoquercetin acts as a regulator of cerebral ischemic injury and thus may exhibit potential in the development of an interventional therapy for cerebral ischemic stroke.

When cerebral ischemia and reperfusion occur, ROS are generated, and can cause the brain to become damaged (17). A previous study has demonstrated that isoquercetin acts via an antioxidative mechanism to protect against cerebral ischemic damage in vivo (18). The protective effects of isoquercetin may involve the direct scavenging of free radicals and/or other endogenous antioxidant mechanisms. The results of the present study demonstrate that isoquercetin decreased the levels of ROS and LDH release. Previous studies have shown that the neuroprotective effects of isoquercetin are mediated by an anti-apoptotic signaling pathway involving the deactivation of ERK, an increase in the protein expression of Nrf2 and the inhibition of apoptosis $(19,20)$.

Marked changes in ERK are associated with the pathophysiology of focal cerebral ischemia. Previous studies have shown that ischemia and the subsequent neuronal damage are associated with inhibition of ERK1/2, and the activation of ERK1/2 has neuroprotective effects $(21,22)$. Furthermore, it has been demonstrated that OGD/R treatment of hippocampal cultures leads to the activation of ERK1/2 (23). Under oxidative stress conditions, Nrf2 is liberated from repression by 
Kelchlike ECH-associated protein 1, and then translocates to the nucleus, where it interacts with small Maf proteins to form a heterodimer, identifies and binds to a cis-acting antioxidant response element, and, finally, assembles the whole transcription machinery for transcription of its target genes (24). When activated, for example by pharmacological agents including resveratrol, curcumin and retinoic acid, Nrf2 signaling protects tissue from oxidative and cytotoxic injury, and is a key pathway for protecting tissue against endogenous and exogenous stress (25). The results of the present study reveal that isoquercetin notably increases the expression of Nrf2 in OGD/R-treated neurons. It has previously been shown that Nrf2 overexpression is positively regulated via ERK1/2, and that blocking ERK1/2 activation inhibits hyperoxia-induced Nrf2-activation (22).

In the present study, neurotoxicity was found to be reduced by isoquercetin in an ERK1/2-dependent manner, and the isoquercetin-induced activation of ERK1/2 was blocked by the ERK1/2 inhibitor PD98059. It remains to be determined whether the finding of an ERK1/2-dependent and -independent component of isoquercetin inducible Nrf2 is an artifact of blocking an isoquercetin preferred signaling pathway. The present study also suggested that isoquercetin suppressed ROS and LDH release in hippocampal neurons via the regulation of ERK1/2-mediated Nrf2 expression, which may provide a useful strategy for the moderation of neurodegenerative diseases, including Parkinson's disease and Alzheimer's disease.

In conclusion, the present study has demonstrated that isoquercetin is an effective antioxidant that protects against OGD/R, induces reductions in ROS and LDH levels, and increases Nrf2 expression via the activation of ERK1/2, leading to a significant reduction in cell death. However, additional studies exploring the biological activities of isoquercetin in vivo are required to support the use of isoquercetin as a potential drug for MCAO.

\section{Acknowledgements}

The present study was supported by the following three projects: (1) the 2013-2014 National Clinical Key Specialty Construction Project, supported by the National Health and Family Planning Commission, China; (2) The Early Management and Process Optimization Study of Acute Ischemic Stroke in ER, supported by Shanghai Health and Family Planning Commission, China (grant no. 201440496) and (3) the Shanghai Advanced Integrated Traditional Chinese And Western Medicine Personnel Training Project, supported by Shanghai Health and Family Planning Commission, China (grant no. ZY3 RCPY 4 2009).

\section{References}

1. Nabel EG and Braunwald E: A tale of coronary artery disease and myocardial infarction. N Engl J Med 366: 54-63, 2012.

2. Go AS, Mozaffarian D, Roger VL, Benjamin EJ, Berry JD, Blaha MJ, Dai S, Ford ES, Fox CS, Franco S, et al: Heart disease and stroke statistics-2014 update: A report from the American Heart Association. Circulation 129: e28-e292, 2014.

3. Lee JH, Shin HK, Park SY, Kim CD, Lee WS and Hong KW: Cilostazol preserves CA1 hippocampus and enhances generation of immature neuroblasts in dentate gyrus after transient forebrain ischemia in rats. Exp Neurol 215: 87-94, 2009.

4. Chan PH: Reactive oxygen radicals in signaling and damage in the ischemic brain. J Cereb Blood Flow Metab 21: 2-14, 2001
5. Zhou J, Yoshitomi H, Liu T, Zhou B, Sun W, Qin L, Guo X, Huang L, $\mathrm{Wu} \mathrm{L}$ and Gao M: Isoquercitrin activates the AMP-activated protein kinase (AMPK) signal pathway in rat H4IIE cells. BMC Complement Altern Med 14: 42, 2014.

6. Liu Z, Zhang A, Guo Y and Dong C: Electrochemical sensor for ultrasensitive determination of isoquercitrin and baicalin based on DM- $\beta$-cyclodextrin functionalized graphene nanosheets. Biosens Bioelectron 58: 242-248, 2014.

7. Morand C, Manach C, Crespy V and Remesy C: Quercetin 3-O-beta-glucoside is better absorbed than other quercetin forms and is not present in rat plasma. Free Radic Res 33: 667-676, 2000.

8. Tu XK, Yang WZ, Wang CH, Shi SS, Zhang YL, Chen CM, Yang YK, Jin CD and Wen S: Zileuton reduces inflammatory reaction and brain damage following permanent cerebral ischemia in rats. Inflammation 33: 344-352, 2010.

9. Longa EZ, Weinstein PR, Carlson S and Cummins R: Reversible middle cerebral artery occlusion without craniectomy in rats. Stroke 20: 84-91, 1989.

10. Wu XM, Qian ZM, Zhu L, Du F, Yung WH, Gong Q and $\mathrm{Ke} \mathrm{Y}$ : Neuroprotective effect of ligustilide against ischemia-reperfusion injury via up-regulation of erythropoietin and down-regulation of RTP801. Br J Pharmacol 164: 332-343, 2011.

11. Joshi CN, Jain SK and Murthy PS: An optimized triphenyltetrazolium chloride method for identification of cerebral infarcts. Brain Res Brain Res Protoc 13: 11-17, 2004.

12. Alrob OA, Sankaralingam S, Ma C, Wagg CS, Fillmore N, Jaswal JS, Sack MN, Lehner R, Gupta MP, Michelakis ED, et al: Obesity-induced lysine acetylation increases cardiac fatty acid oxidation and impairs insulin signalling. Cardiovasc Res 103: 485-497, 2014

13. Rodrigues Hell RC, Silva Costa MM, Goes AM and Oliveira AL: Local injection of BDNF producing mesenchymal stem cells increases neuronal survival and ynaptic stability following ventral root avulsion. Neurobiol Dis 33: 290-300, 2009.

14. Poulose SM, Carey AN and Shukitt-Hale B: Improving brain signaling in aging: Could berriesbe the answer? Expert Rev Neurother 12: 887-889, 2012.

15. Dajas F, Rivera F, Blasina F, Arredondo F, Echeverry C, Lafon L, Morquio A and Heinzen H: Cell culture protection and in vivo neuroprotective capacity of flavonoids. Neurotox Res 5: 425-432, 2003.

16. Ossola B, Kääriäinen TM and Männistö PT: The multiple faces of quercetin in neuroprotection. Expert Opin Drug Saf 8: 397-409, 2009.

17. Allen CL and Bayraktutan U: Oxidative stress and it srole in the pathogenesis of ischaemic stroke. Int J Stroke 4: 461-470, 2009.

18. Boots AW, Haenen GR and Bast A: Health effects of quercetin: From antioxidant to nutraceutical. Eur J Pharmacol 585: 325-337, 2008.

19. Kilic U, Kilic E, Matter CM, Bassetti CL and Hermann DM: TLR-4 deficiency protects against focal cerebral ischemia and axotomy-inducedneurodegeneration. Neurobiol Dis 31: 33-40, 2008.

20. Loniewski KJ, Patial S and Parameswaran N: Sensitivity of TLR4- and -7-induced NF kappa B1 p105-TPL2-ERK pathway to TNF-receptor-associated factor6 revealed by RNAi in mouse macrophages. Mol Immunol 44: 3715-3723, 2007.

21. Hu HH, Li SJ, Wang P, Yan HC, Cao X, Hou FQ, Fang YY, Zhu XH and Gao TM: An L-type calcium channel agonist, bay $\mathrm{K} 8644$, extends the window of intervention against ischemic neuronal injury. Mol Neurobiol 47: 280-289, 2013.

22. Liu RL, Xiong QJ, Shu Q, Wu WN, Cheng J, Fu H, Wang F, Chen JG and Hu ZL: Hyperoside protects cortical neurons from oxygen-glucose deprivation-reperfusion induced injury via nitric oxide signal pathway. Brain Res 1469: 164-173, 2012.

23. Motohashi $\mathrm{H}$ and Yamamoto M: Nrf2-Keap1 defines a physiologically important stress response mechanism. Trends Mol Med 10: 549-57, 2004.

24. Shen G, Hebbar V, Nair S, Xu C, Li W, Lin W, Keum YS, Han J, Gallo MA and Kong AN: Regulation of Nrf2 transactivation domain activity. The differential effects of mitogen-activated protein kinase cascades and synergistic stimulatory effect of Raf and CREB-binding protein. J Biol Chem 279: 23052-23060, 2004.

25. Pitha-Rowe I, Liby K, Royce D and Sporn M: Synthetic triterpenoids attenuate cytotoxic retinal injury: Cross-talk between Nrf2 and PI3K/AKT signaling through inhibition of the lipid phosphatase PTEN. Invest Ophthalmol Vis Sci 50: 5339-5347, 2009. 